\title{
Diet-Nutrition and Cancer Prevention (Review)
}

\author{
Ruchit Khanna', Anand Kumar², Ruchika Khanna \\ ${ }^{1}$ Department of Pharmacology, Maharishi Markandeswar University, Mulana, Ambala, Haryana, India, ${ }^{2}$ Department \\ of Oral and Maxillofacial Surgery, Career Post Graduate Institute of Dental Sciences, Lucknow, Uttar Pradesh, \\ India, ${ }^{3}$ Department of Oral Medicine and Radiology, Teerthanker Mahaveer Dental College and Research Center, \\ Moradabad, Uttar Pradesh, India
}

\begin{abstract}
When nutrients are isolated from whole foods, they do not always act the same. Several nutrients found in fruit and vegetables show an inverse association with oral cancer. Nutrition has enhanced more bring into focus on the levels of biochemical sequences through which ingredients are digested, and other living organic structure are converted from one form to another via metabolism and metabolic pathways. Nutrition also centers on in what way diseases, conditions, and dilemmas can be averted or diminished with a healthy diet. Cancer is preceded by pre-cancerous lesions. Numerous investigations have attempted to determine whether consuming particular food increases or decreases a person's chance of acquiring cancer. Sadly, various thoughts have conflicted issues, so it is troublesome to understand what impact foods or dietary supplements have on cancer risk. In summation, nutrition includes recognizing how particular disorders, conditions or dilemmas may be generated by dietary factors, such as inadequate food (malnutrition), food allergies, metabolic diseases, etc.
\end{abstract}

Key words: Cancer, diet, health, nutrition

\section{INTRODUCTION}

Hippocrates (Greece, 460BC-370BC), one nutrient theory - according to Hippocrates everybody is the same, no matter what they have been eating, or where they have lived. He concluded that every food must contain one nutrient which makes us the way we are. This one-nutrient myth continued for thousands of years. Hippocrates is also famous for having said "Let thy food be thy medicine and thy medicine be thy food. [1]" Poor diets and low physical activity levels are among the main cause of many acute and chronic disease including obesity and excess risks of non-

\begin{tabular}{|c|c|}
\hline \multicolumn{2}{|c|}{ Access this article online } \\
\hline Publisher & \multirow[b]{2}{*}{$\begin{array}{l}\text { Website: } \\
\text { http://www.renupublishers.com }\end{array}$} \\
\hline & \\
\hline & $\begin{array}{l}\text { DOI: } \\
\text { 10.5958/2394-4196.2014.00004.1 }\end{array}$ \\
\hline
\end{tabular}

communicable diseases, including hypertension, cardiovascular disease, type 2 diabetes, stroke and certain cancers.

Cancer arises with a variation in the structure of DNA. DNA equips the cells with an essential set of instructions, such as when to develop and replicate. A variation in DNA structure is perceived as a mutation; moreover it can change the instructions that control mechanism of cell growth. This indicates that cells proceed to improve rather than freezing when they should. This prompts the cells to reproduce in a stubborn method, creating a lump of tissue called tumors.

The rapidly increasing, abundance of published studies reveals that specific groups of food ingredients create the impression to help prevent cancer and at times also support the body in fighting the disease if already own cancer. These elements are called as chemo-protective agents, involving a group commonly known as antioxidants; they resemble to defend the body from

\footnotetext{
Address for correspondence:

Dr. Anand Kumar, Career Post Graduate Institute of Dental Sciences, 17/12 Indira Nagar, Lucknow - 226 016, Uttar Pradesh, India. E-mail: anandkmr901@gmail.com
}

Submission: 14 Oct 2014; Revision: 06 Nov 2014; Acceptance: 10 Dec 2014 
the consequences of carcinogenic (cancer-causing) elements.

Diet can also influence oral health via copious mechanisms. Dietary deficiencies are identified to provoke numerous diseases that reveal as oral changes. In reckon, specific foods have the one and the other beneficial and disease-causing ability, possibly harming the teeth, periodontal structures, and mucosa.

The American Cancer Society publishes nutrition and physical activity guidelines to advise health care professionals and the general public about dietary and other lifestyle practices that reduce cancer risk. ${ }^{[2]}$

\section{ANTI-OXIDANTS IN PREVENTING CANCER}

Antioxidants are substances that interact with and neutralize free radicals, thus inhibiting them from producing harm. Antioxidants are also known as "free radical scavengers."

The body makes some of the antioxidants it uses to neutralize free radicals. These antioxidants are called endogenous antioxidants. However, the body relies on external (exogenous) sources, primarily the diet, to obtain the rest of the antioxidants it needs. These exogenous antioxidants are commonly called dietary antioxidants. Fruits, vegetables, and grains are rich sources of dietary antioxidants. Some dietary antioxidants are also available as dietary supplements. ${ }^{[3-5]}$

There are hundreds, presumably thousands, of various substances that can function as antioxidants. The utmost common ones are vitamin $\mathrm{C}$ - vitamin $\mathrm{E}$, beta-carotene, and other related carotenoids - along with the mineral's selenium and manganese. They are joined by glutathione, coenzyme Q10, lipoic acid, flavonoids, phenols, polyphenols, phytoestrogens, and numerous more.

Researchers have examined in case receiving dietary antioxidant supplements can help reduce the chance of developing or dying from cancer in humans.

Other anti-oxidants, which are not vitamins, are additionally seen in fruits and vegetables and resemble to have highest cancer-fighting features. Beta carotene, a substance which provides bit vegetables their appearance as carrots and peppers, and which the body transforms into vitamin $\mathrm{A}$, is one of the aforementioned. Just as soon as ingested in an original form (food rich in beta-carotene, such as green vegetables, carrots, or peppers), such a thing may enhance immune responses in the body by inciting a particle that supports the immune system target and suppress cancer cells.

\section{EFFECTS OF VEGETABLES AND FRUITS}

Greater consumption of vegetables, fruits, or both together has been associated in the majority of epidemiological studies with a lower risk of lung, oral, esophageal, stomach, and colon cancer. Evidence is less strong for cancers considered hormonal, such as breast and prostate. Diet can be an important factor even in cancers caused, in large part, by other factors. For instance, many studies have found a lower risk of lung cancer among those who eat more vegetables and/or fruits in their diet. ${ }^{[6-8]}$

Although the major factor that causes lung cancer is tobacco smoking, diet also modifies risk, both in smokers and in lifelong nonsmokers. Vegetables and fruits are complex foods, each containing more than 100 potentially beneficial vitamins, minerals, fiber, and other substances that may help to prevent cancer. Vegetables and fruits also contain specific phytochemicals, such as carotenoids, flavonoids, terpenes, sterols, indoles, and phenols that show benefit against certain cancers in experimental studies.

Although not food, alcohol intake has also been shown to be associated with the development of oral cancer, particularly when it is used in combination with smoking. The theory is that the alcohol dehydrates the mucosal cell walls, increasing permeation of the smoke carcinogens into the mucosa. Excessive alcohol use may also be associated with a lowering of the body's ability to use antioxidants in fighting oral cancer development. ${ }^{[9]}$

\section{Water and Other Fluids}

Consumption of water and other fluids may decrease the risk of bladder cancer, as water thinned out the concentration of carcinogens and reduces the period in which they are in touch with the bladder lining. Drinking at minimum eight cups of fluid a day is usually suggested, and some investigations show that even more may be beneficial. ${ }^{[10]}$ 
Tea

Green tea contains antioxidant molecules called catechins that appear very beneficial. Some researchers have proposed that tea protect against cancer because of its antioxidant content. In animal research, some teas (including green tea) have been proved to decrease cancer risk, but epidemiological investigations have had mixed findings. At present, tea has not been proven to reduce cancer risk in humans. ${ }^{[1]}$

\section{Fiber}

Dietary fiber, additionally acknowledged as roughage or bulk, involes all parts of plant foods in that human body cannot ingest or absorb. Unlike different food ingredient, of this kind as fats, proteins or carbohydrates which human body breaks into metabolites and digests - fiber is not absorbed by body. Preferably, it passes nearly intact through stomach, small intestine, and colon and out of body. A considerable collection of research recommends that eating a variety of foods accommodating high fiber has a shielding influence against colon cancer. Dietary fiber includes an extensive variety of plant carbohydrates that are not absorbable by humans. Soluble fiber supports to decrease blood cholesterol including, it helps reduce the chance of coronary heart disease. Large sources of fiber are vegetables, whole grains, beans, together with fruits. Associations between fiber and cancer risk are uncertain, but consumption of these foods is still prescribed because they include other substances that can help prevent cancer and because of their other health benefits. ${ }^{[12]}$

\section{Bioengineered Foods}

Bioengineered foods are prepared by combining genes from different plants or organisms to develop a plant's resistance to insect pests, retard waste, or promote transportability, flavor, nutrient composition, or other desired properties. In the hypothesis, these combined genes might generate substances that could produce adverse reactions among sensitized or allergic individuals. Despite, there is currently no evidence in order that the substances located in bioengineered foods presently on the market are toxic or that they would either enhance or reduce cancer risk on account of the added genes. ${ }^{[12]}$ Still there is a lack of data that can demonstrate that bioengineered foods have any effect on cancer risk.

\section{Heredity}

Destruction to the genes that regulate cell division and maturation can both inherit or acquired during one's lifetime. Specific varieties of mutations or a genetic disturbance can heighten the chance of cancer. Nutrients in the diet can shield DNA from being destroyed. Physical activity, weight control, and diet might slow or hinder the spread of cancer in people with enhanced genetic risk for cancer. The many communications between diet and genetic factors are an essential and complicated topic of broad current investigation concern. ${ }^{[13]}$

\section{Garlic}

Garlic is utilized for flavoring in cuisine and is novel on account of its large sulfur content. It also contains arginine - oligosaccharides, flavonoids, and selenium - each and every of which may be useful for health. The health benefits of the allium compounds included in garlic, and other vegetables in the onion group have been publicized extensively, frequently because garlic has very little outcomes on blood cholesterol levels. Garlic is currently under study for its efficacy to diminish cancer risk. Inadequate evidence exists at this point to hold a precise function for this vegetable in cancer prevention. In particular, large human studies that seem to be on diet and cancer have proposed in that people who eat more garlic possess a lower chance of stomach, prostate, mouth and throat, kidney, and colorectal cancer. The impact on risk of breast, bladder, ovarian, and lung cancers is not so much as obvious. Garlic is currently under research for its ability to diminish cancer risk. ${ }^{[13]}$

\section{Saccharin}

Saccharin has remained approximately for over 100 years and pretends to be the "best researched sweetener." High doses of the artificial sweetener saccharin provoke the development of bladder stones that can begin to bladder cancer was found in research on rats. Saccharin consumption does not produce the development of bladder stones in humans. If the saccharin increased cancer risk in humans, it would do so at doses many times greater than amounts typically consumed by people. ${ }^{[14]}$ Large epidemiological research have not reported progress in bladder cancers among people using saccharin, and saccharin has been excluded from the list of established human carcinogens by the US National Toxicology Program. ${ }^{[15]}$ 
The ultimate mechanism of carcinogenesis in rats secondary to sodium saccharin administration seems to be the formation of a calcium phosphate precipitate in the urine (Cohen et al. 1995). ${ }^{[16]}$

\section{Polycyclic Aromatic Hydrocarbons (PAHs)}

PAH compounds are generally produced during incomplete combustion of organic matter. These compounds are also usually found in tobacco smoking. ${ }^{[17]}$ Benzopirene is the best-characterized PAH compound available from the diet. Several animal and human studies suggest that dietary PAH is distributed to organ besides the locally exposed tissues, so it is plausible to consider that dietary PAH may contribute to lung or breast cancer risk, for example. ${ }^{[18]}$

\section{Phytochemicals}

Phytochemicals are naturally occurring, biologically active chemical compounds in plants. The prefix "phyto" is from the Greek word meaning plant. In plants, phytochemicals act as a natural defense system for host plants and provide color, aroma, and flavor. ${ }^{[19]}$ More than 4000 of these compounds have been discovered to date. Phytochemicals are rich sources of vitamins and antioxidants, which are thought to have anti-cancer properties. They are divided into flavonoids, carotenoids, indoles, isothiocyanates, polyphenols, capsaicin, phytoestrogens, phytosterols, protease inhibitors, saponins, sufides, tannins, and terpenes. ${ }^{[20,21]}$

\section{CONCLUSION}

Carcinogenesis can be generated by a variety of substances, both genotoxic and non-genotoxic. Treatment for cancer can generate outcomes, incorporating weight loss, lethargy, nausea, diarrhea, constipation, decreased blood counts and heightened risk of infection. The Indicated consequences can hinder one's capability to stay nourished and interfere with treatment.

It may be essential to employ a feeding tube throughout the time that sustaining oral cancer treatment to assure that an individual is receiving sufficient nutrition. A feeding tube may be implanted as a substitute for short-term or long-term basis. They can help keep fit and served during treatment, and can be dropped in after treatment if needed. The tube can be effortlessly eliminated as quickly as natural eating is feasible. These are the principal sources of the nutritious ingredients which a body feel the necessity for sustaining a healthy and competent immune system, the primary defense against cancer and other illnesses. One who has escaped from carcinoma, many times have questions about food preferences, physical exercise, and dietary supplements aid to promote and recover their quality of life and survival.

\section{REFERENCES}

1. Ding YS, Ward J, Hammond D, Watson CH. Mouth-level intake of Benzo[a]pyrene from reduced nicotine cigarettes. Int J Environ Res Public Health 2014;11:11898-914.

2. Weinhouse S, Bal DG, Adamson R, Dwyer J, Kleinman RE, Kritchevsky D, et al. American Cancer Society guidelines on diet, nutrition, and cancer. The Work Study Group on Diet, Nutrition, and Cancer. CA Cancer J Clin 1991;41:334-8.

3. Bouayed J, Bohn T. Exogenous antioxidants-double-edged swords in cellular redoc state: Health beneficial effects at physiologic doses versus deleterious effects at high doses. Oxid Med Cell Longev 2010;3(4):228-37.

4. Davis CD, Tsuji PA, Milner JA. Selenoproteins and cancer prevention. Annu Rev Nutr 2012;32:73-95.

5. Patterson RE, White E, Kristal AR, Neuhouser ML, Potter JD. Vitamin supplements and cancer risk: The epidemiologic evidence. Cancer Causes Control 1997;8:786-802.

6. Giovannucci E. Tomatoes, tomato-based products, lycopene and cancer: Review of the epidemiologic literature. J Natl Cancer Inst 1999;91:317-31.

7. Biesalski HK, Bueno de Mesquita B, Chesson A, Chytil F, Grimble R, Hermus RJ, et al. European consensus statement on lung cancer: Risk factors and prevention. Lung Cancer Panel. CA Cancer J Clin 1998;48:167-76.

8. The effect of vitamin $\mathrm{E}$ and beta carotene on the incidence of lung cancer and other cancers in male smokers. The Alpha-Tocopherol, Beta Carotene Cancer Prevention Study Group. N Engl J Med 1994;330:1029-35.

9. Omenn GS, Goodman GE, Thornquist MD, Balmes J, Cullen MR, Glass A, et al. Effects of a combination of beta carotene and vitamin A on lung cancer and cardiovascular disease. N Engl J Med 1996;334:1150-5.

10. Jia WH, Luo XY, Feng BJ, Ruan HL, Bei JX, Liu WS, et al. Traditional Cantonese diet and nasopharyngeal carcinoma risk:A large-scale case-control study in Guangdong, China. BMC Cancer 2010;10:446.

11. Ashley MJ, Ferrence R. Moderate drinking and health: The scientific evidence. Contemp Drug Probl 1994;21:1-204.

12. Katiyar SK, Mukhtar H. Tea in chemoprevention of cancer: Epidemiologic and experimental studies. Int J Oncol 1996;8:221-38.

13. Byers T, Nestle M, McTiernan A, Doyle C, Currie-Williams A, Gansler T, et al. American Cancer Society guidelines on nutrition and physical activity for cancer prevention: Reducing the risk of cancer with healthy food choices and physical activity. CA Cancer J Clin 2002;52:92-119.

14. World Cancer Research Fund in Association with American Institute for Cancer Research. Food, Nutrition and the Prevention of Cancer: A Global Perspective. Washington DC: World Cancer Research Fund; 1997.

15. National Research Council. Carcinogens and Anticarcinogens in the Human Diet. Washington, DC: National Academy Press; 1996.

16. Cohen SM, Cano M, Garland EM, St John M, Arnold LL. Urinary and urothelial effects of sodium salts in male rats. Carcinogenesis 1995;16:343-8. 
17. Tyagi P, Shrama G, Hussain S, Kundu A, Gaur A, Kumar P. Magnanimous effects and role of dietary nutrition on carcinogenesis: A comprehensive review of literature. Int Med J Sifa Univ 2014;1:5-8.

18. Goldman R, Shields PG. Food mutagens. J Nutr 2003;133:965S-73.

19. Khambete N, Kumar R. Carcinogens and cancer preventors in diet. Int J Nutr Pharmacol Neurol Dis 2014;4:4-10.

20. Johary A, Jain V, Misra S. Role of lycopene in the prevention of cancer. Int J Nutr Pharmacol Neurol Dis 2012;2:167-70.

21. Mullaicharam AR, Maheshwaran A. Pharmacologic effects of curcumin. Int J Nutr Pharmacol Neurol Dis 2012;2:92-9.

How to cite this article: Khanna R, Kumar A, Khanna R. Diet-Nutrition and Cancer Prevention (Review). Int J Dent Med Spec 2014;1(2):33-37.

Source of Support: None; Conflict of Interest: None 